\title{
A New Mechanism of the Nanoindentation Process
}

\section{Gerasimov $A^{* 1}$, Vepkhvadze $\mathrm{M}^{1}$, Gorgadze $\mathrm{K}^{1}$, Buachidze $\mathrm{D}^{1}$, Chiradze $\mathrm{G}^{2}$ and Metonidze $\mathrm{M}^{1}$}

${ }^{1}$ Department of Physics, Georgian Technical University, Tbilisi, Georgia

${ }^{2}$ Akaki Tsereteli State University Department of Physics Kutaisi, Georgia

*Corresponding author: Gerasimov A, Department of Physics, Georgian Technical University, Tbilisi, Georgia, Tel: +995 555357221, E-mail: aleksi.gerasimovi@gmail.com

Citation: Gerasimov A, Vepkhvadze M, Gorgadze K, Buachidze D, Chiradze G, et al. (2018) A New Mechanism of the Nanoindentation Process. J Mater Sci Nanotechnol 6(1): 103

Received Date: December 28, 2017 Accepted Date: February 22, 2018 Published Date: February 26, 2018

\begin{abstract}
A new mechanism of the nanoindentation process based on the consideration of the decrease the energy of the chemical bonds as a result of the pressure of the indenter on the material is proposed. This leads to an increase the mobility of the atoms, which in the beginning creates point defects and as the external force increases, melting and material destruction occurs.
\end{abstract}

Keywords: Nanoindentation; Elastoplastic; Antibonding bands

\section{Introduction}

Nanoindentation is one of the most significant methods for studying the mechanical properties of solids and thin films in submicron regions and surface layers [1]. The process of nanoindentation consists of elastic and elastoplastic deformations. In this process, a very important point is the transition of the elastic-to elastoplastic deformation. There are several hypotheses concerning the nature of this abrupt transition, the most important of which are the homogeneous generation of a dislocation under the indenter [2-5] and the plasticity mechanism, realized through the diffusion mobility of nonequilibrium point defects [6-9]. But these hypotheses cannot explain the fact, that in a precession study of the dependence of the penetration depth of an indenter on a sample from a load, regions with an abrupt increase in the penetration depth of the indenter with a constant load value are observed (Figure 1) [1].

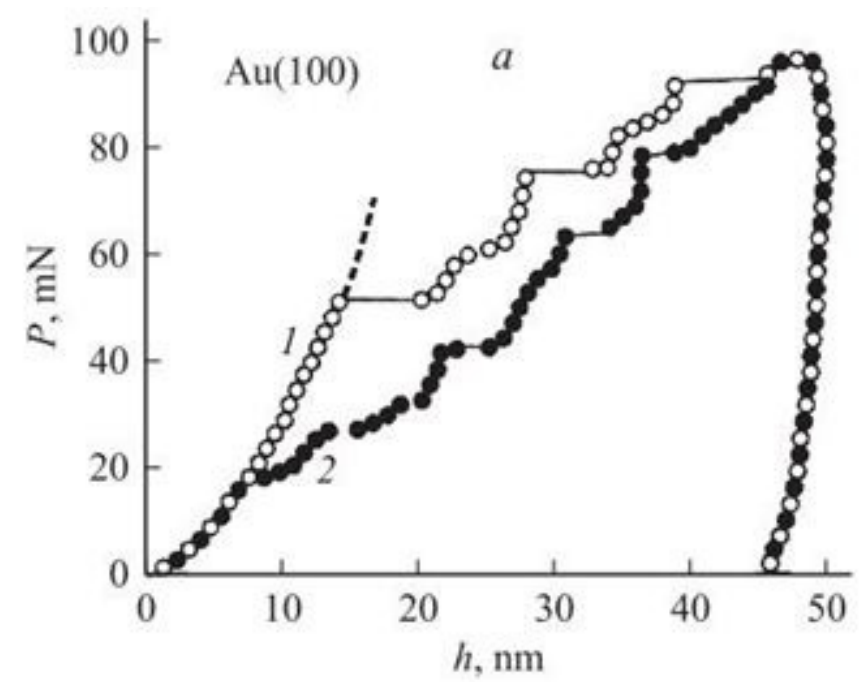

Figure 1: Diagram $P$ - $h$ ( $P$ is the load on the indenter, and $\mathrm{h}$ is the depth of its penetration). Single crystal gold: 1 - atomically smooth surface, 2 - surface with $2 \mathrm{~nm}$ steps [1]

"Despite a great body of experimental data collected to date, complete clarity in the mechanisms of loss of elastic stability under nanocontact deformation has not yet been achieved" [1]. In addition, during nano-and microindentation, an increase in the 
hardness of the material is observed with a decrease in the depth of penetration of the indenter into the material (Figure 2) which occurs when the load decreases [1]. The nature of this dependence is still not clear [1].

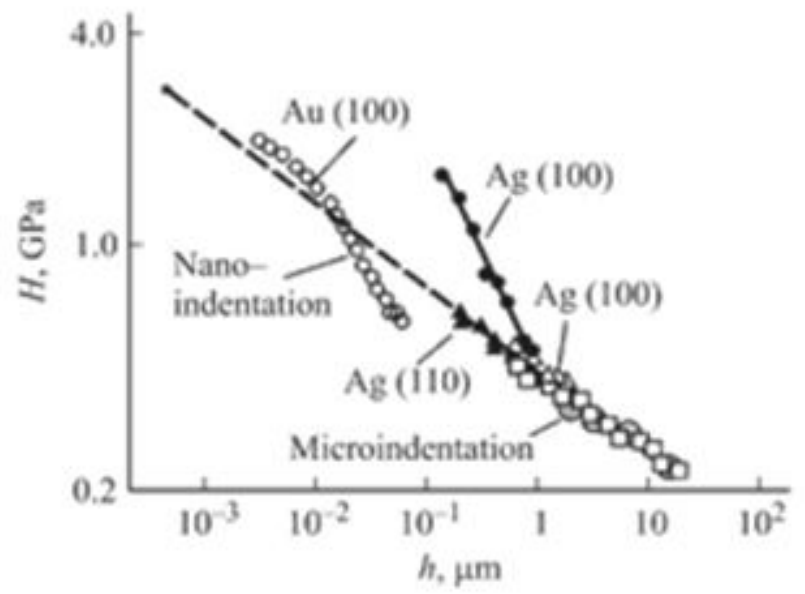

Figure 2: Hardness vs. penetration depth of the indenter [1]

\section{Results and Discussion}

In this paper, new mechanisms of the above phenomena are proposed. These mechanisms are based on new ideas about the role of chemical bonds in the motion of atoms (molecules) in condensed media [10,11] described in the molecular-potential theory [12]. In contrast to the molecular-kinetic theory, according to which [13] for the atom (molecule) to change its location in the condensed media, a kinetic energy fluctuation near the given atom must be formed to overcome the potential barrier (Figure 3 ), the molecular-potential theory [12] requires the appearance of potential energy fluctuations near a given atom to reduce the chemical bond energy, which corresponds to a decrease in the height of the potential barrier (Figure 1) to be overcome by the atom (molecule) [10-12]. It should also be borne in mind that in condensed media the electrons participating in the creation of a chemical bond can be in two quantum states.

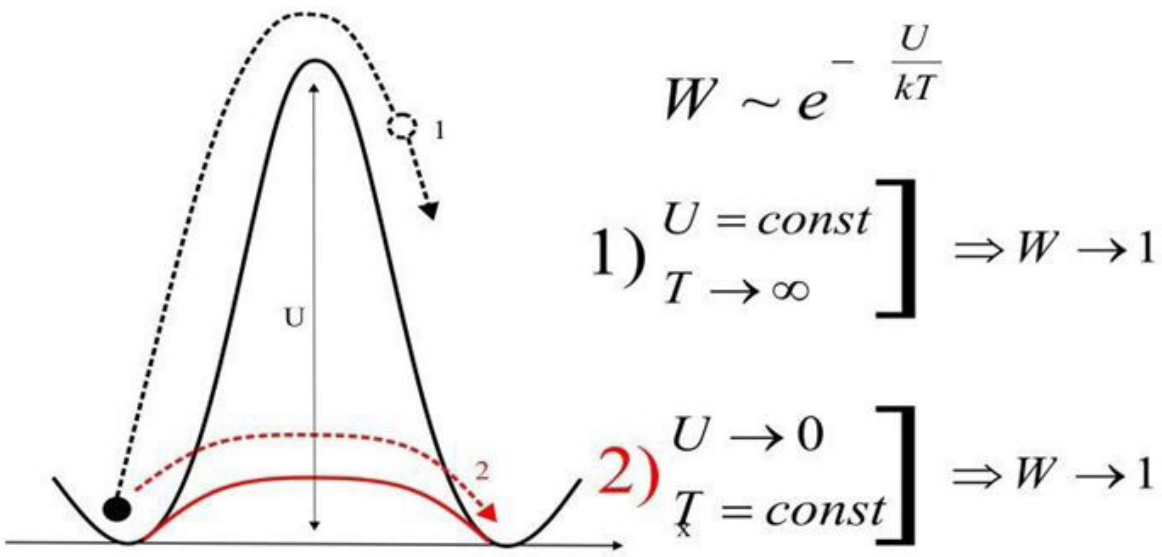

Figure 3: (1) According to the molecular-kinetic theory, for the atom to move in a solid, it, as a result of fluctuations, must obtain a kinetic energy sufficient to overcome the potential barrier $U$. The probability of such a process is described by the above dependence, where $W$ is the fluctuation generation probability for a given atom, $T$ is the absolute temperature; $k$ is the Boltzmann constant (the barrier height $U$ is assumed constant for a given substance). (2) According to the new ideas, for the atom to move, it is necessary to break chemical bonds by some athermal method, which means the decrease in the interatomic potential energy to a minimum: $U \rightarrow 0$

Being in one of the quantum states, they increase the energy of the chemical bond (bonding states), whereas in the other they reduce it (antibonding states) [14]. The decrease in the energy of the chemical bond occurs due to the appearance of antibonding quasiparticles - excited free electrons and holes - near a given atom. In accordance with the theory of molecular orbitals of chemical bonding extended to solids and liquids [14], the energy spectrum of electrons consists of bonding and antibonding bands (Figure 4). In semiconductors, these bands are separated by a band gap, while in metals they are overlapped. The presence of an electron in the bonding band increases, and its absence (hole) decreases the chemical bond. The strength of the chemical bond of a given atom (molecule) with neighboring atoms is determined by the difference between the bonding and antibonding electrons located near it. The smaller this difference, the weaker the chemical bond, and when the difference is zero, the chemical bond disappears 
(sublimation, evaporation, boiling) [10-12].

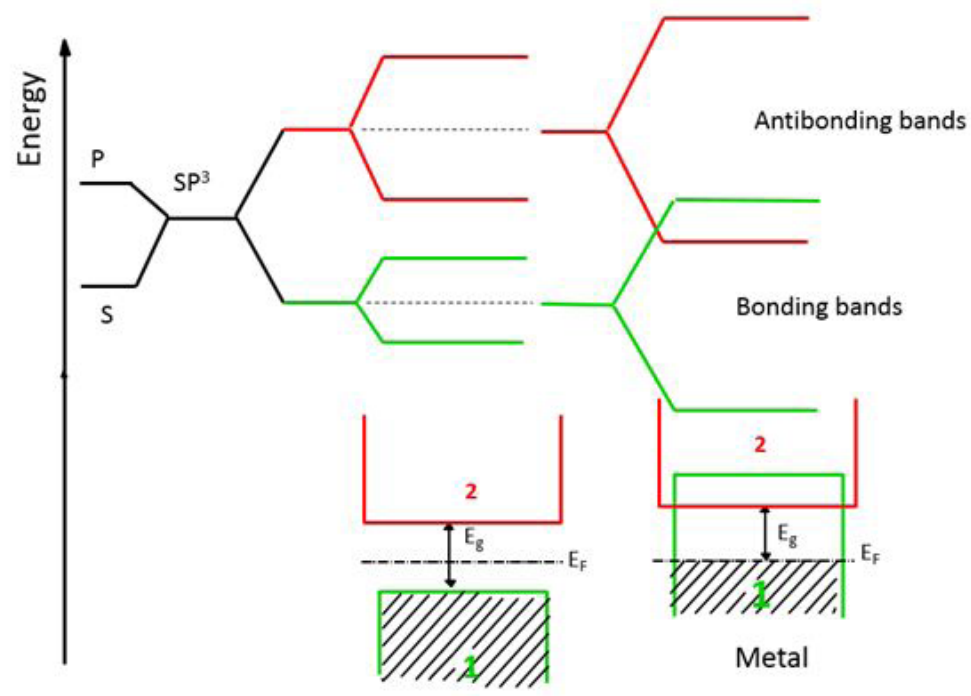

Semiconductor

Figure 4: Formation of hybridized orbitals, their splitting into bonding and antibonding orbitals, and as the atoms converge, occurrence of the bonding and antibonding bands, respectively. e.g., is a band gap or a pseudo band gap

Thus, the transfer of an electron from the bonding to antibonding band (in metals, transitions occur between the Fermi level and the bottom of the antibonding band - this energy distance is referred to as pseudo band gap) (Figure 4) means a decrease in the chemical bond of the substance (i.e., if it is a solid body, it should become softer and increase in size, which is proved experimentally). The transfer of electrons can be accomplished in various ways (Figure 5). In any case, the motion of the atom will be facilitated. In its chaotic motion, an electron and a hole can be brought near a certain atom with a probability of $\mathrm{n} / \mathrm{Na}$ ( $\mathrm{n}$ is the concentration of antibonding quasiparticles, and $\mathrm{Na}$ is the total concentration of the atoms of the substance) in different $\beta$ quantities (potential energy fluctuation) with probability $(n / \mathrm{Na})$ and weaken differently the chemical bond. The greater the concentration of antibonding quasiparticles at a given temperature, the greater the probability of the atomic motion and the more intense the observed process will be. And when the critical concentration of antibonding quasiparticles is reached, $n_{k}=\mathrm{Na} m^{*} / \mathrm{Ma}$ ( $m^{*}$ is the effective mass of the quasi-free electron, $M a$ is the mass of the atom (molecule) of the substance), the melting process begins, regardless of the manner the antibonding quasiparticles have been generated [10-12].

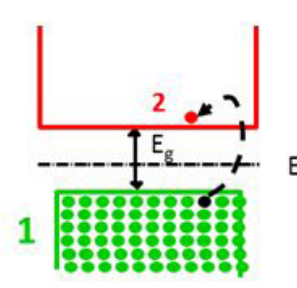

Temperature

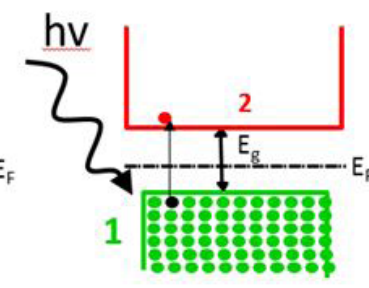

Light

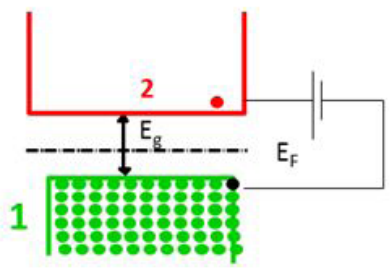

Injection

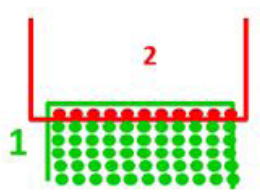

Pressure

Figure 5: If under the influence of temperature, light, injection, pressure, the electrons will be transferred from the bonding to antibonding bands, the chemical bond in the substance will be weakened

The molecular-potential theory made it possible to explain all the phenomena observed in the effects of hardness changes under the influence of light (photomechanical effect-PME), electric current or field (electromechanical effect-EME), temperature (thermomechanical effect-TME), magnetic field (magnetomechanical effect-MME) [10,12], i.e., their dependence on the intensity and spectral composition of light, temperature, type and concentration of impurities, etc.

When the nanoindenter contacts the surface of the studied sample, there occur sufficiently high pressures that change the energy spectrum of the electrons participating in chemical bonding, which is manifested as a change in the relative position of the energy bands on the energy scale [15-17]. In different crystallographic directions, the bands shift differently, but in some direction, the bonding and antibonding bands always approach each other, and the concentration of antibonding quasiparticles increases at constant temperature. In the area of contact between the indenter and the sample, a local decrease in the distance between the bands [15] and an increase in the concentration of antibonding quasiparticles (Figure 6) take place, which facilitates the motion of atoms (molecules), i.e., the formation of point defects. However, when the critical concentration of antibonding quasiparticles is reached, local melting occurs, which leads to an abrupt increase in the depth of penetration of the indenter under the influence 
of a constant load. This, of course, increases the contact area, resulting in a decrease in pressure and, accordingly, in concentration of antibonding quasiparticles, and the melting process stops. Therefore, to further increase the depth of indenter penetration, the load must be increased (Figure 1). With the further increase in the load, the situation described above may occur and a new abrupt increase in the penetration depth of the indenter (Figure 1), etc., may take place.

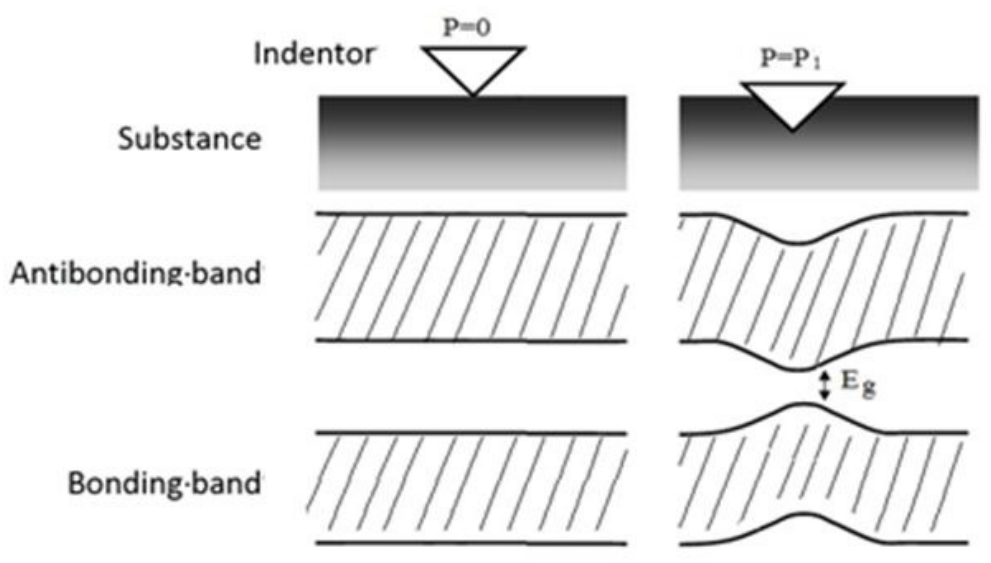

Figure 6: Schematic representation of a local decrease in the energy distance between the bonding and antibonding bands under the influence of the indenter pressure

It is clear that the load intensity at which the abrupt increase in the penetration depth of the indenter occurs will depend on the nature and processing of the material, the geometry of the indenter tip, the loading rate and the temperature of the experiment that will affect the formation of antibonding quasiparticles. With increasing temperature, thermal transitions of electrons will also contribute to the achievement of a critical concentration of antibonding quasiparticles. Therefore, an abrupt increase in the depth of penetration of the indenter will occur at lower loads (Figure 7), which is observed experimentally [9]. With an increase in the loading rate, the equilibrium critical concentration of antibonding quasiparticles does not have time to be reached, for which purpose an increase in the load is required, which is observed experimentally [1]. The experimental data on the trixotropic effect also confirm the mechanism of an abrupt increase in the depth of penetration of the indenter under the action of a constant load due to local melting: the appearance of humps of an amorphous substance in the material at the indenter penetration interface which in its structure is very similar to a solid frozen after melting $[7,18,19]$.

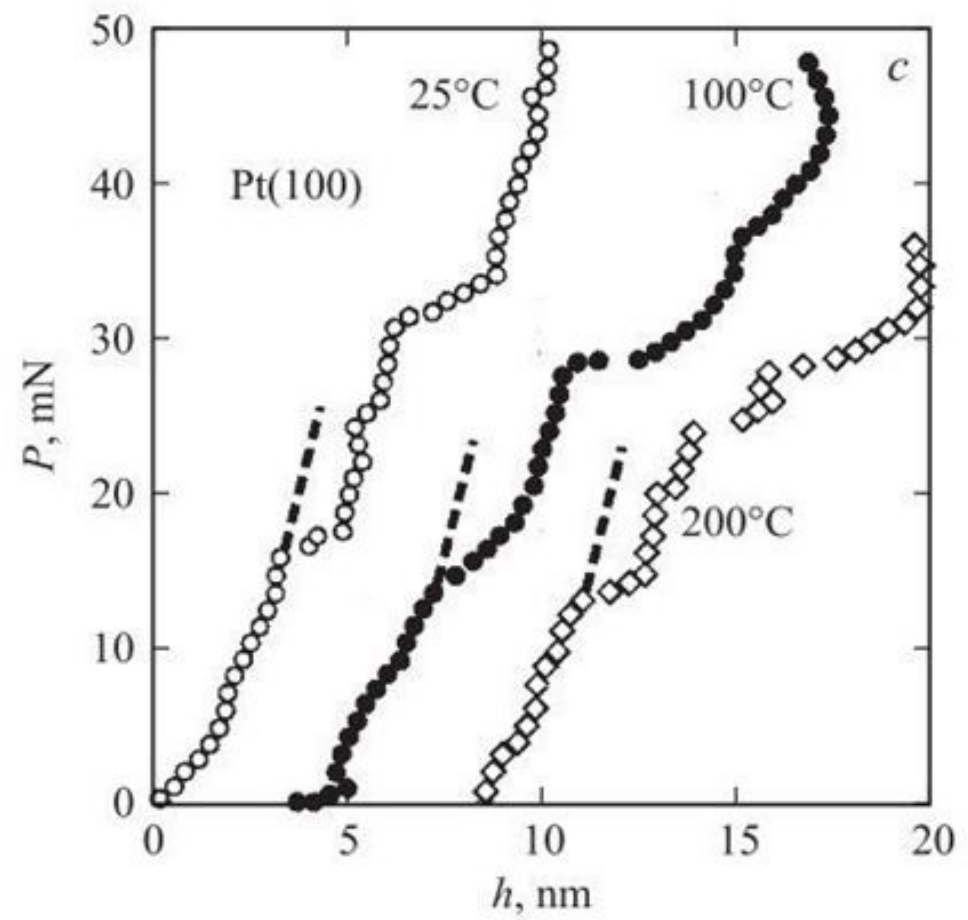

Figure 7: S Temperature dependence of the $P$ - $h$ diagram $(P$ is the load on the indenter, and $\mathrm{h}$ is the depth of its penetration) for platinum [1] 
To explain the dependence of hardness on the depth of indentation, let us consider how the strength of chemical bonds varies in the direction from the surface to the volume. Extreme atoms on the surface have unsaturated chemical bonds - the excessive electrons which bind to the neighboring side atoms, and a phenomenon of dimerization type is observed [20]. This excessive negative charge density flows over the chemical bonds of atoms located deep from the surface (Figure 8), decreasing with depth the faster, the smaller and the dielectric constant of the substance [21].

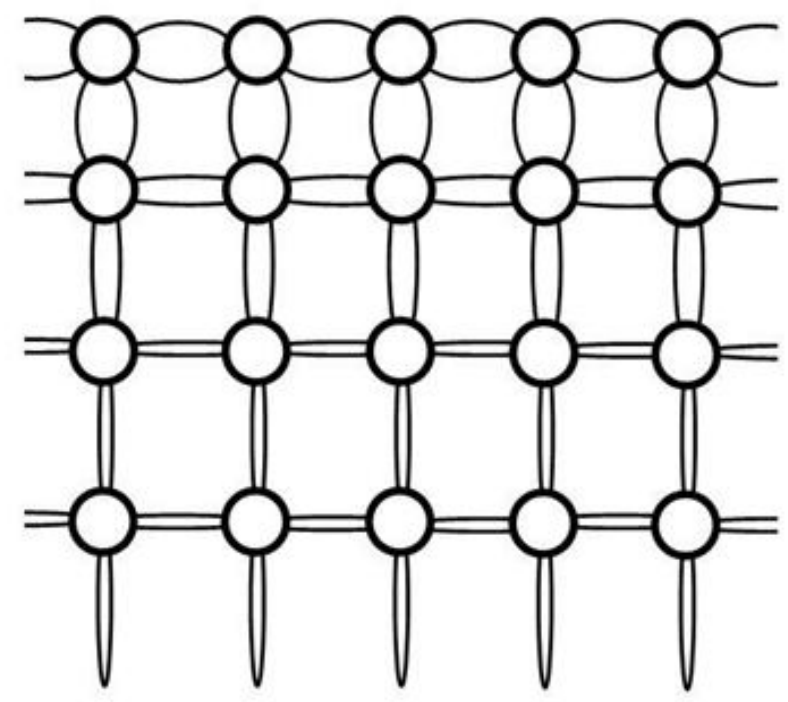

Figure 8: Schematic representation of the distribution of the excessive negative charge density over the chemical bonds of atoms located deep from the surface

Thus, an increase in the negative charge density towards the surface increases the strength of the chemical bonds and, consequently, the hardness (Figure 2), which is observed experimentally [9]. This mechanism is proved by the fact that if we somehow increase the concentration of antibonding quasiparticles on the surface reducing thereby the strength of the chemical bonds, so that the concentration antibonding quasiparticles on the surface of the sample be maximum and fall down deep into the bulk of the sample, the hardness on the surface must be lower than in the depth. We carried out this experiment [22], and the results are shown in Figure 9. In the indentation process, the surface of the studied sample was illuminated in one case by strongly absorbable monochromatic light and in the other-by white light penetrating to a considerable depth. As can be seen from the figure, in the first case, the hardness on the surface, where the concentration antibonding quasiparticles is maximum, is lower than at the depth, where antibonding quasiparticles are not generated by light. The dependence of the increase in hardness deep from the surface coincides with the decrease in the concentration of antibonding quasiparticles generated by light. In the second case, light, penetrating to the entire studied depth of the sample, generates antibonding quasiparticles fairly uniformly, and a decrease in hardness occurs evenly throughout the whole depth of the sample. These data, in our opinion, prove unambiguously the correctness of the proposed mechanism.

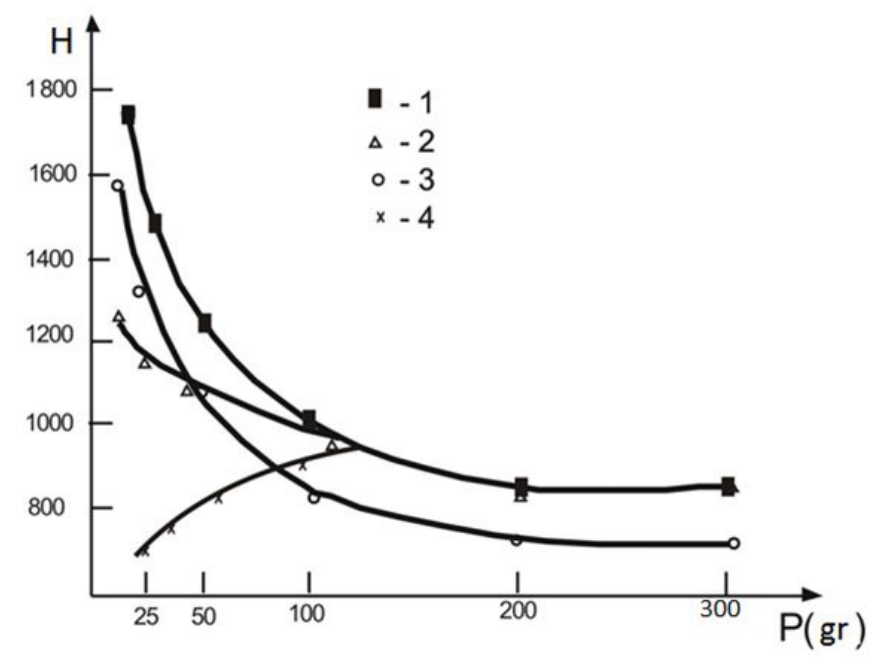

Figure 9: Si hardness vs. the load applied to the indenter; the increase in the load means the increase in the depth of immersion of the indenter: (1) - in the dark; (2) - under laser illumination $(h v>E g)$, when the concentration of antibonding quasiparticles is created only in the surface layer; (3) - when illuminated by white light passing through the Si filter $(h v<E g)$, when antibonding quasiparticles are created in the entire volume; $(4)$ - when illuminated by a high intensity laser, i.e., at a high concentration of antibonding quasiparticles, the hardness value on the surface is lower than in the volume 


\section{Conclusion}

Thus, it has been shown that under the action of an external force, in this case an indenter, the bonding and antibonding zones converge and the formation of antibonding quasiparticles - excited free electrons and holes, which reduces the energy of chemical bonds. This makes it easier to change the location of the atoms, which in the beginning creates point defects and as the external force increases, the material melts and breaks down [23].

\section{References}

1. Golovin YI (2008) Nanoindentation and mechanical properties of solids in submicrovolumes, thin near-surface layers, and films. Phys solid State 50: 2205-36.

2. Golovin YuI, Dub SN (2003) A Spasmodic Transition from Elastic to Elastoplastic Deformation at the Initial Stage of Nanoindentation. Trabsactions (Doklady) Rus Acad Sci 393: 108.

3. Kramer DE, Yoder KB, Gerberich WW (2001) Surface constrained plasticity: oxide rupture and the yield point process. Philosophical Magazine A 81: 2033-58.

4. Van Vliet KJ, Li Ju, Zhu T, Yip S, Suresh S (2003) Quantifying the early stages of plasticity through nanoscale experiments and simulations. Phys Rev B 67: 104105.

5. Mason JK, Lund AC, Schuh CA (2006) Determining the activation energy and volume for the onset of plasticity during nanoindentation. Phys Rev B 73: 054102.

6. Indenbom VL (1970) Interstitial (Crowdion) Mechanism of Plastic Deformation and Failure. Pis'ma v JhETF (JETF Letters) 12: 526.

7. Rozhanskii VN, Nazarova MP, Svetlov IZ, Kalashnikova LK (1970) Dislocation and Crowdion Plasticity of Corundum at Room Temperature. Phys Status Solidi 41: 579 .

8. Golovin YuI, Tyurin AI, (1994) Interstitial mechanisms for plastic low in the initial stage of loading during microindentations. Pis'ma v JhETF (JETF Letters) 60: 722 .

9. Golovin YuI, Tyurin AI, Farber BY (2002) Investigation of time-dependent characteristics of materials and micromechanisms of plastic deformation on a submicron scale by a new pulse indentation technique. J Mater Sci 37: 895-904.

10. Gerasimov (2005) A Principles of a low-temperature technology of fabrication of semiconductor devices. Tbilisi State University, Tbilisi, Georgia.

11. Gerasimov AB (1991) Electron-hole mechanism of migration and defect interaction. Proc 4th Int Conf Mater Sci Forum 65-66: 47-52.

12. Gerasimov AB (2012) Fundamentals of the molecular-potential theory. 2nd Int Conf Nanotechnol Nano Tbilisi, Georgia.

13. Frenkel YaI (1945) Kinetic theory of liquids. Moscow-Leningrad.

14. Harrison U (1983) Electronic structure and the properties of solids. Mir Pub, Moscow.

15. Polyakova AL (1979) Deformation of semiconductors and semiconductor devices. Moskva Nauka 168.

16. Polyakova AL (1972) Physical principles of the operation of semiconducting transducers sensitive to mechanical quantities; Soviet Physics-Acoustic 18: 1.

17. Gerasimov A, Kvesitadze G, Vepkhvadze M (2016) Chemical Bonds in the Processes of Plasticity, Fluidity and Mechanical Destruction of Materials. Bull Georg Natl Acad Sci 10: 98.

18. Golovin YuI, Tyurin AI (1995) On the dynamics and mechanism of the initial stage of immersion of an indenter with microindentation of crystals. Fiz Tverd Tela 37: 1562 .

19. Rodríguez de la Fuente O, Zimmerman JA, González MA, de la Figuera J, Hamilton JC, et al. (2002) Dislocation Emission around Nanoindentations on a (001) fcc Metal Surface Studied by Scanning Tunneling Microscopy and Atomistic Simulations. Phys Rev Lett 88: 036101.

20. Bekhstedt F, Enderlein R (1988) Semiconductor surfaces and interfaces. Mir Pub, Moscow.

21. Grimmeiss HG (1977) Deep-level impurities in semiconductors. Ann Rev mater Sci 7: 341-76.

22. Gerasimov AB, Chiradze GD, Kutivadze NG (2001) Investigation of the physical nature of the photomechanical effect. Fizika I Tekhnika Poluprovodnikov (FTP) 35: 1-70.

23. Chiradze G, Gerasimov A, Kvesitadze G, Vepkhvadze M (2016) Chemical Bonds in Changing the Hardness of Nanomaterials. Bull Georg Natl Acad Sci 10: 99-104. 